\title{
Fluorescent emission in different silicon carbide polytypes
}

\section{S. Castelletto, B. C. Johnson, I. Aharonovich, A. Parker}

S. Castelletto, B. C. Johnson, I. Aharonovich, A. Parker, "Fluorescent emission in different silicon carbide polytypes," Proc. SPIE 8923, Micro/Nano Materials, Devices, and Systems, 89230B (7 December 2013); doi: $10.1117 / 12.2033265$

SPIE Event: SPIE Micro+Nano Materials, Devices, and Applications, 2013, Melbourne, Victoria, Australia 


\title{
FLUORESCENT EMISSION IN DIFFERENT SILICON CARBIDE POLYTYPES
}

\author{
S. Castelletto ${ }^{1 *}$, B.C. Johnson ${ }^{2,3}$, I. Aharonovich ${ }^{4}$ and A. Parker ${ }^{1}$ \\ ${ }^{1}$ School of Aerospace, Mechanical \& Manufacturing Engineering \\ RMIT University, Bundoora, VIC, Australia \\ ${ }^{2}$ School of Physics, The University of Melbourne, VIC, Australia 1 \\ ${ }^{3}$ CQC2T, University of Melbourne, Parkville, VIC 3010, Australia \\ ${ }^{4}$ School of Physics and Advanced Materials, University of Technology Sydney, Ultimo, \\ NSW 2007, Australia \\ *Corresponding author email: stefania.castelletto@rmit.edu.au
}

\begin{abstract}
Silicon carbide $(\mathrm{SiC})$ is a widely used material in several industrial applications such as high power electronics, light emitting diodes, and in research application such as photo-voltaic and quantum technologies. As nanoparticles it can be synthetised in many sizes and different polytypes from $200 \mathrm{~nm}$ down to $1 \mathrm{~nm}$. In the form of quantum dots they are used as optical biomarkers, and their emission, occurring from the blue to the orange spectral region, is based on quantum confinement effect. In this work we report on emission in the red and near infrared in different SiC polytypes, specifically in $4 \mathrm{H}, 6 \mathrm{H}$ and $3 \mathrm{C}$. In $4 \mathrm{H} \mathrm{SiC}$ the red visible emission yielded non classical light attributed to an intrinsic defect, identified as a carbon-antisite vacancy pair. Similar spectral emission was observed in 3C SiC bulk and nanoparticles, also yielding very bright single photon emission. Emission in the far red has been observed in homogeneous hetero-structure in $\mathrm{SiC}$ tetrapods.
\end{abstract}

Keywords: optical defects, silicon carbide nanoparticles, non-classical photo-emission, confocal microscopy

\section{INTRODUCTION}

$\mathrm{SiC}$ is a compound of silicon and carbon that exists in many different crystal types (well over 200 polytypes), and as a semiconductor with a wide-band gap (depending on the polytypes), it has an high thermal conductivity, the ability to sustain high electric fields before breakdown, and the highest maximum current density, making it ideal for high power electronics ${ }^{1}$. Such an engineer-friendly material used since many years, has also recently attracted attention in emerging research fields such as quantum computing ${ }^{1-3}$, spintronics ${ }^{4}$ and single photon generation ${ }^{5}$, as several of its intrinsic defects carrying an electron spin can be used as "quantum bits" and more recently they have been isolated providing single photon emission at room temperature.

Its relevance and promises to provide a platform for quantum technology rely on $\mathrm{SiC}$ high quality and ultra-pure large scale production and ready available nanofabrication methods in a large variety of technologies.

At the nanoscale $\mathrm{SiC}$ nanoparticles possess several similarities with other semiconductors quantum dots. For a review on quantum properties in silicon carbide see ref [6].

This paper will review very recent research by the authors in bulk and nanostructures SiC, indicating that this material could result in an advanced platform for the integration of active single defect (quantum system) in existing device technologies (photonics, electronic devices and nano-mechanical resonators) with the exceptional possibility to operate at room temperature.

Among SiC many polytypes only three are of technologically importance because can be produced as high quality, bulk single crystal substrates and films. Two of these polytypes have an hexagonally arranged bilayer configuration with a 4 and 6 bilayer periodicity, referred to as $4 \mathrm{H}-\mathrm{SiC}$ and $6 \mathrm{H}-\mathrm{SiC}$, respectively. The structure of the third polytype is cubic of the zinc-blende type and is referred to as $3 \mathrm{C}-\mathrm{SiC}$. The $4 \mathrm{H}$ and $6 \mathrm{H}-\mathrm{SiC}$ polytypes can be readily produced in electronicgrade wafers suitable for epitaxial growth. Creating high quality $3 \mathrm{C}-\mathrm{SiC}$ wafer-type substrates has been significantly more challenging, although, production of large-area $3 \mathrm{C}$-SiC wafers has been demonstrated. Here we will show recent results on photoluminescence studies in $4 \mathrm{H}, 6 \mathrm{H}$ and $3 \mathrm{C} \mathrm{SiC}$.

Micro/Nano Materials, Devices, and Systems, edited by James Friend, H. Hoe Tan, Proc. of SPIE Vol. 8923, 89230B - (c) 2013 SPIE · CCC code: 0277-786X/13/\$18 - doi: 10.1117/12.2033265 


\section{Bright single photon emission in ultra-pure $4 \mathrm{H} \mathrm{SiC}$}

A range of deep level defects give rise to radiative recombination in $\mathrm{SiC}$. Defect-induced photoluminescence (PL) in $\mathrm{SiC}$ can be detected from the UV to the infrared with some PL lines coinciding with the first and second telecommunication windows. The binary compound nature and the existence of non-equivalent lattice sites in different polytypes gives rise to a large variety of possible defects. Combinations of simple point defects are possible, of recent interest di-vacancies $\left(\mathrm{V}_{\mathrm{Si}} \mathrm{V}_{\mathrm{C}}\right)^{3}$ and $\mathrm{C}$ vacancy-antisite pairs $\left(\mathrm{V}_{\mathrm{C}} \mathrm{C}_{\mathrm{Si}}\right)^{5}$.

(a)

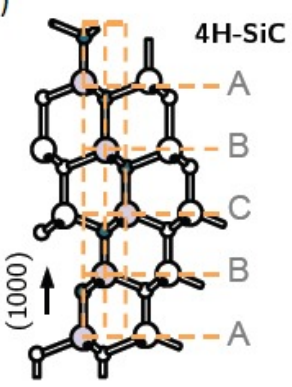

(b)

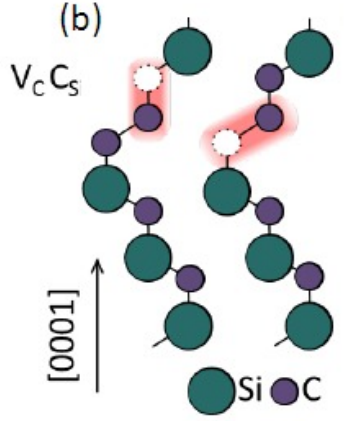

(c)

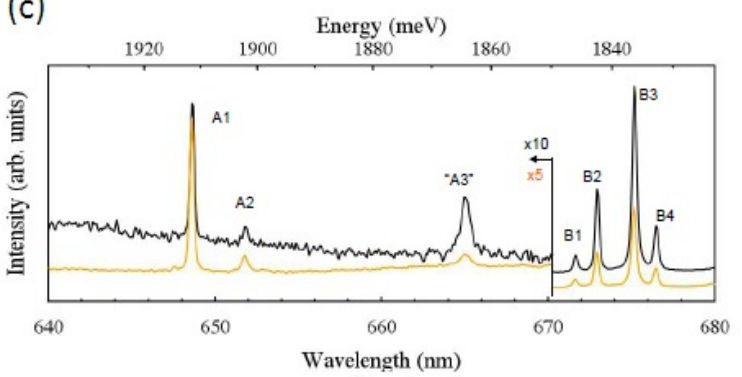

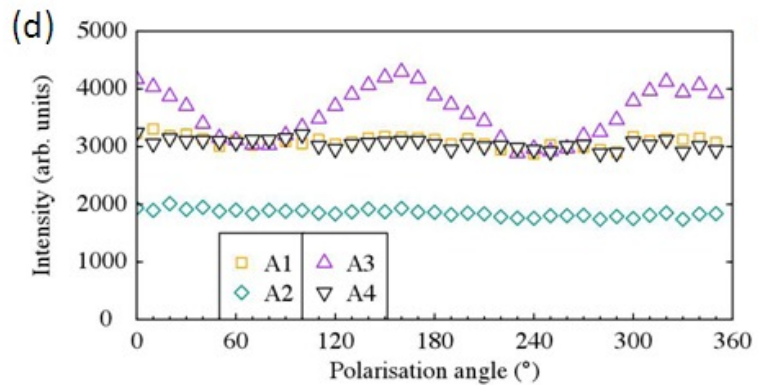

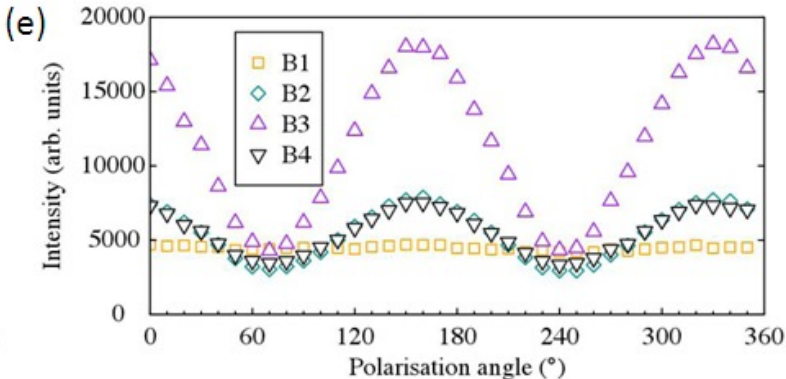

Figure 1 (a) $4 \mathrm{H} \mathrm{SiC}$ atoms layout. (b)The proposed atomic structure of the defects $C_{S i} V_{C}$, giving rise to the photoluminescence, is shown in the (1000) plane (small and large circles represent $C$ and Si atoms; the small open circles are vacancies).

(c)The AB photoluminescence lines observed in an electron-irradiated sample measured at $80 \mathrm{~K} .(\mathrm{d}, e)$, The emission polarization dependence of the $A B$ lines with the excitation beam perpendicular to the $c$ axis.

Before the full use of these defects can be realized in applications relative to quantum technologies or quantum sensing, a number of challenges need to be addressed. Spin manipulation has so far been carried out only for ensembles of defects ${ }^{3}$. A first step towards the application of $\mathrm{SiC}$ defects in quantum technologies is to observe single photon emission from $\mathrm{SiC}$ defects. Such single photon sources have been observed for the first time operating at room temperature. ${ }^{5}$ These particular defects are $C$ anti-site-vacancy pairs, firstly identified in ensemble ${ }^{7}$, giving rise to the AB PL system ${ }^{8}$. The single photon statistics for emission from a single $\mathrm{C}$ anti-site-vacancy pair and the defect creation in ultra-pure $4 \mathrm{H} \mathrm{SiC}$ are described in ref [5]. In Figure 1 (a) 4H Silicon Carbide structure is shown together with the specific defect atomistic structure (b). This defect is the brightest single photon emitter in a solid state system operating at room temperature, indicating a quantum efficiency of about $70 \%$. C anti-site-vacancy pair occurs in the region 650-700 nm due to the existence of eight Zero Phonon Lines (ZPLs) associated to different location of the defect in the lattice, known as axial and basal sites. PL emission of the various ZPLs is shown in Figure 1 (c) at low temperature for all AB lines, while Figure 1 (d) and (e) show the polarization of the A and B lines which is in agreement with the modelled symmetry of the defect $^{5}$. At room temperature a single line results broadened.

$4 \mathrm{H} \mathrm{SiC}$ samples were carefully irradiated with electrons at high energy $(2 \mathrm{MeV})$ and various fluences (from $10^{13}$ to $10^{17}$ $\mathrm{e} / \mathrm{cm}^{2}$ ) to create vacancies and then annealed at various temperatures (from 300 to $800{ }^{\circ} \mathrm{C}$ ). We performed confocal microscopy to identify bright spots on the confocal maps using $532 \mathrm{~nm}$ and $660 \mathrm{~nm}$ excitation laser. We verified single photon emission using a Hanbury-Brown and Twiss interferometer and analysed the photo-luminescence (PL) at room temperature ${ }^{5}$. Ensemble PL measurements and PL polarization were performed at low temperature.

\section{PL emission in $4 \mathrm{H}$ and $6 \mathrm{H} \mathrm{SiC} \mathrm{devices}$}

$\mathrm{SiC}$ pn junction diodes in both $4 \mathrm{H}$ and $6 \mathrm{H}$ polytypes have been fabricated ${ }^{11}$. The optically active defects in these devices are investigated using both photoluminescence (PL) and electroluminescence (EL) techniques. 
Various $\mathrm{p}+\mathrm{n}$ juncton diodes were formed with $4 \mathrm{H}$ and $6 \mathrm{H}$ SiC n-type epilayers grown by hot-wall chemical vapour deposition. Defects in the as-prepared devices and those introduced by implantation and electron irradiation are investigated. To characterise the defects a Renishaw MicroRaman Spectrometer was used for the Raman scattering, PL and EL measurements. A $532 \mathrm{~nm}$ laser was directed through a 20x long working distance objective with a 0.4 NA onto the sample. The visible wavelength range detector is a fan cooled Si CCD while the NIR detector is a LN2 cooled InGaAs photodiode array.

To understand the evolution of defects in $\mathrm{SiC}$ and to optimize their incorporation into $\mathrm{SiC}$ devices an annealing study was first performed on $4 \mathrm{H} \mathrm{SiC}$ irradiated with $2 \mathrm{MeV}$ electrons to a fluence of $1 \times 10^{17} \mathrm{~cm}^{-2}$. The annealing kinetics of the $\mathrm{C}_{\mathrm{Si}} \mathrm{V}_{\mathrm{C}}$ defect is compared with that of the $\left(\mathrm{V}_{\mathrm{Si}}\right)$ and the divacancy $\left(\mathrm{V}_{\mathrm{C}} \mathrm{V}_{\mathrm{Si}}\right)$. The $\mathrm{V}_{\mathrm{Si}}$ defect which has ZPL emission around 861.5 and $916.3 \mathrm{~nm}$ is the most unstable and the PL decreases dramatically around $600^{\circ} \mathrm{C}$. The $\mathrm{C}_{\mathrm{Si}} \mathrm{V}_{\mathrm{C}}$ defect has eight ZPLs in the $648-675 \mathrm{~nm}$ range and increases in intensity until $800^{\circ} \mathrm{C}$ after which it anneals out. The $\mathrm{V}_{\mathrm{C}} \mathrm{V}_{\mathrm{Si}}$ defects with ZPLs in the NIR are the most stable and peaked in intensity at $1000^{\circ} \mathrm{C}$ but were not annealed out in the annealing range considered.

Figure 2 shows the PL spectra of the as-prepared SiC devices. All devices showed Raman features and current voltage characteristics indicative of a high quality device. However, broad PL features were observed in the 700-1000 nm range. EL was also observed while the devices were under bias (not shown).

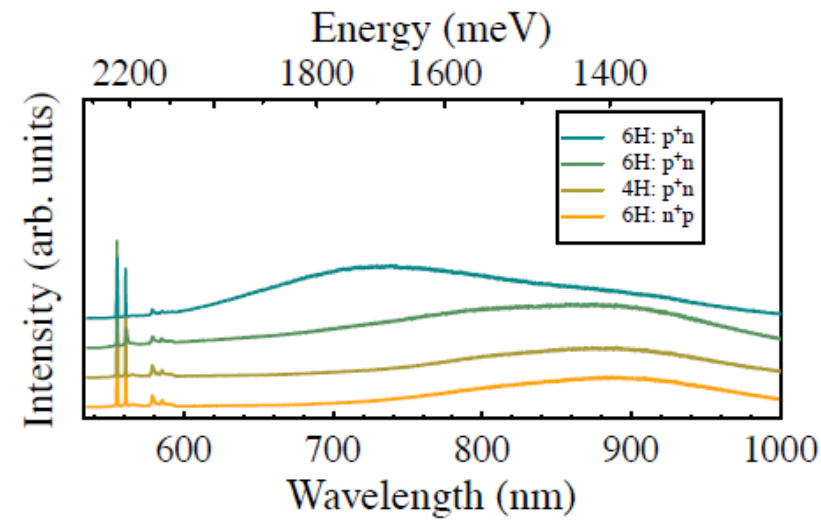

Figure 2- PL spectra of the 6H-SiC devices at room temperature.

\section{3C SiC nanoparticles}

Semiconductor nanocrystals have enabled or transformed research in technologically important areas including biolabelling, solid state lighting, and quantum information processing.

At the nanoscale, $\mathrm{SiC}$ shows quantum confinement effect and luminescence properties within the 400-460 $\mathrm{nm}$ emission range ${ }^{9}$. SiC nanoparticles can be produced in sizes as small as $1.4 \mathrm{~nm}$ and no protective shells are needed for making SiC QDs biocompatible or for stabilizing them in aqueous systems. The fabrication of colloidal SiC quantum dots has been achieved from milling different polytypes $(3 \mathrm{C}, 6 \mathrm{H}$, and $4 \mathrm{H})$ of bulk $\mathrm{SiC}$ crystals using electrochemical method. The three types of obtained $\mathrm{SiC}$ QDs show quite-similar photoluminescence and photoluminescence excitation properties. The photoluminescence peak (in the visible from $450 \mathrm{~nm}$ to $600 \mathrm{~nm}$ ) depends on the excitation wavelength and their lifetime is in the few ns region.

In this work using confocal microscopy and AFM combined with and Hanbury-Brown interferometer (Figure (d) and (e)), we studied SiC nanoparticle PL, observing a broad band emission at room temperature further in the red region with a peak around $700 \mathrm{~nm}$ in nanoparticles of about $250 \mathrm{~nm}$ size (Figure 3(b)). This emission resulted very bright and in a similar spectral region of the $4 \mathrm{H}$ single photon source defect, therefore it is likely to be attributed to the same defect. Further experimental and theoretical work is needed however to fully identify this defect in $3 \mathrm{C}^{12}$. Single photon emission has been observed (not shown) ${ }^{12}$. As a comparison PL were also studied in bulk n-type $3 \mathrm{C} \mathrm{SiC}$ (Figure $3 \mathrm{c}$ ). 


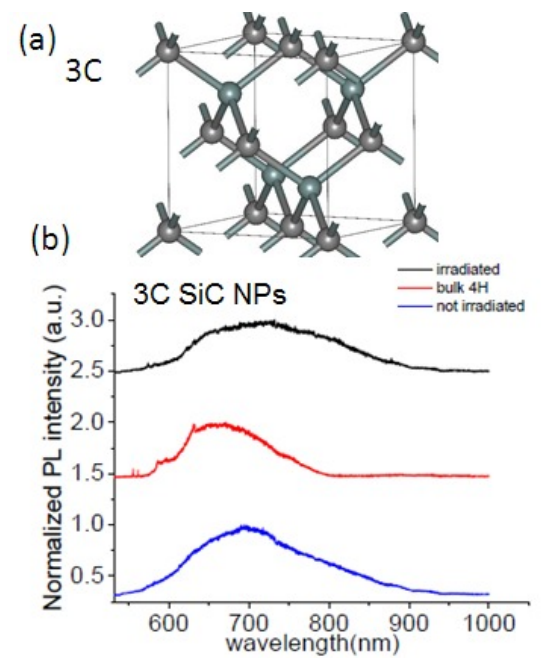

(c)
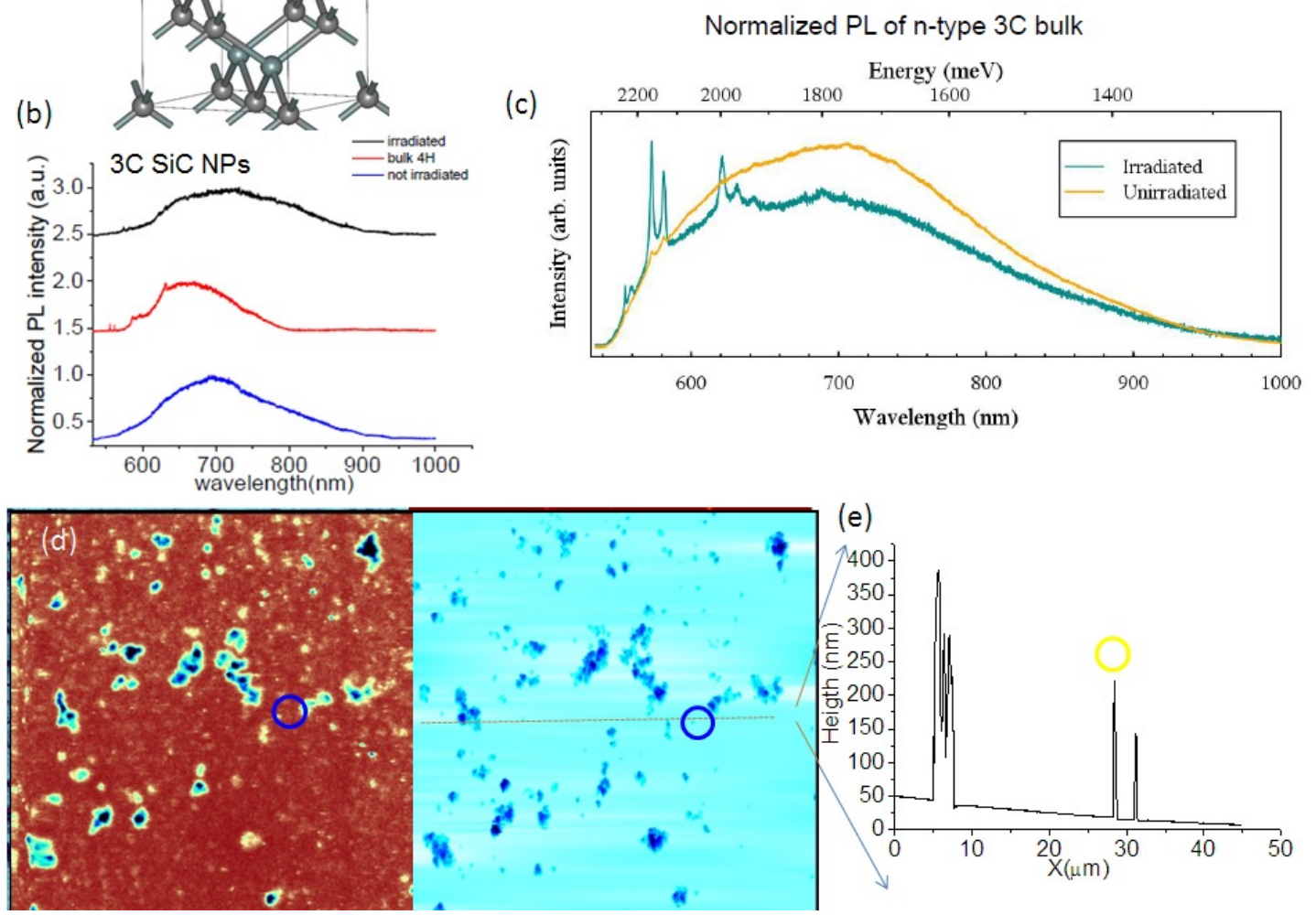

Figure 3- (a) Structure of 3 C SiC from Wikimedia Commons. (b) Normalised PL from oxidised commercial 3C SiC nanoparticles from electron irradiated samples and not irradiated samples. A comparison with emission in bulk $4 H$, yielding single photon emission is shown. (c) Normalised PL from electron irradiated and not irradiated bulk n-type 3C SiC. (d) $45 x 45 \mu^{2}$ confocal and AFM scan of the same area of spin coated $3 C$ SiC nanoparticles (NP) on a cover glass. The circle corresponds to a non-irradiated nanoparticles with PL shown in (b) (e) Cross section providing the height of the SiC NPs studied from the AFM scan.

\section{SiC tetrapods}

The emission properties of quantum dots can be controlled by adjusting their size, altering the material composition, or by building core-shell type structures to improve exciton confinement. Increased structural complexity of a nanostructure can yield even greater control over its excitonic and optical properties.

Another interesting nanostructures in $\mathrm{SiC}$ have recently been synthesized via microwave plasma enhanced chemical vapor deposition from seeding a silicon substrate using adamantane ${ }^{10}$.Tetrapods grown by this technique have four legs, $3 \mathrm{C} \mathrm{SiC}$ is nucleated from adamantane in the $\mathrm{Si}$-rich sol gel environment while the growth environment was carbon-rich, promoting a transition to the growth of $4 \mathrm{H}-\mathrm{SiC}$. The coexistence of $3 \mathrm{C}$ and $4 \mathrm{H}$ in these nanostructures corresponds to homogeneous hetero-structure giving rise to PL in the spectral region between $600 \mathrm{~nm}$ and $800 \mathrm{~nm}$. In this case the emission is consistent with spatially indirect exciton transition from the $3 \mathrm{C}$ core to the $4 \mathrm{H}$ legs, due to a narrower full width half-maximum emission (FHWM) of $5 \mathrm{~nm}$ at room temperature, which cannot be associated defect typically created in $\mathrm{SiC}^{13}$.

Figure 4(a) shows scanning electron microscope image of the tetrapods. The tetrapods exhibit some variation in size and symmetry and do not exhibit a preferred growth orientation. The legs are $40 \pm 10 \mathrm{~nm}$ in diameter and $90 \pm 20 \mathrm{~nm}$ long. Through detailed investigation of the structural and compositional properties of the $\mathrm{SiC}$ tetrapods, it is determined that the tetrapods have $4 \mathrm{H}$ arms and likely grow from a $3 \mathrm{C}$ core.

Remarkably, the tetrapods exhibit narrow band fluorescence as shown in Figure 4(b). The emission maximum varies for different tetrapods, appearing between 550 and $800 \mathrm{~nm}$ and having a FWHM of $\sim 5 \mathrm{~nm}$ - the narrowest emission known 
for any quantum dots or tetrapod structures. The variation in PL is believed to arise from structural differences among the different tetrapods.

Finally, detailed correlation measurements reveal the most of the tetrapods exhibit single photon emission, as confirmed by measuring the photon statistics of the emitted light (not shown). This makes the $\mathrm{SiC}$ tetrapods a prime candidate for future sensing and quantum applications ${ }^{12}$.

(a)

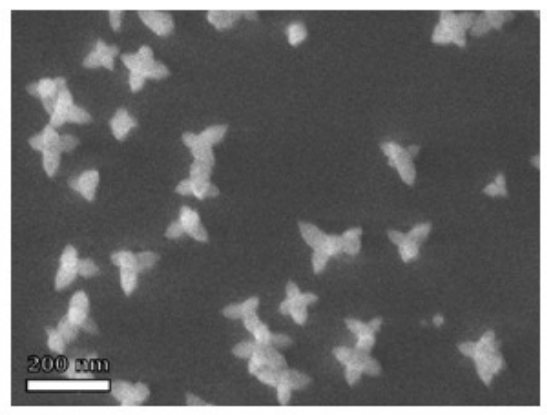

(b)

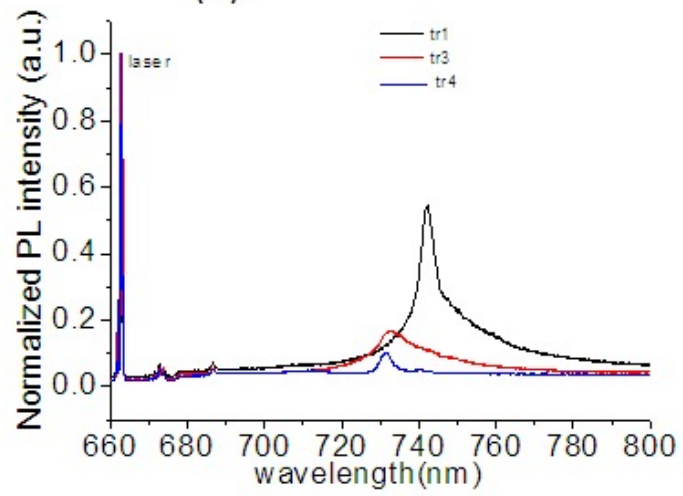

Figure 4. (a) SEM image of SiC tetrapods (b) PL measured on single tetrapods identified using confocal microscopy.

\section{SUMMARY}

Red and near infrared photoluminescence in various polytype of $\mathrm{SiC}$ such as $4 \mathrm{H}, 6 \mathrm{H}$ and $3 \mathrm{C}$ are presented. As striking novelty if compared to the present literature on PL in SiC, under confocal microscopy with single defect sensitivity, we observed a strong PL in all polytypes in the region from $600 \mathrm{~nm}$ to $700 \mathrm{~nm}$, in some cases yielding single photon emission $^{5}$. This PL can be present in as grown material and enhanced in electron irradiated materials. In $4 \mathrm{H} \mathrm{SiC} \mathrm{this}$ emission has been attributed to a defect identified as carbon-antisite vacancy pair as the modelling of this defect agrees with the experimental observation for what concerns its PL polarization properties. Similar broad band emission is observed in 3C nanoparticles and 3C bulk material, with the expected enhanced emission occurring in $200 \mathrm{~nm}$ nanoparticles size. In this polytype the appurtenance of the defect is still debated. Finally emission in the far red and near infrared $(600$ to $800 \mathrm{~nm}$ ) with narrower FWHM at room temperature is observed from $\mathrm{SiC}$ tetrapods. Due to the heterostructure of the tetrapods, made of $3 \mathrm{C}$ and $4 \mathrm{H} \mathrm{SiC}$ with nanoscale sizes, this $\mathrm{PL}$ is motivated by exciton quantum confinement.

Presently $\mathrm{SiC}$ is the most prominent and rich material system being investigated from the point of view of optical deep defects as well as quantum confinement avenues, due to different polytype engineering of nanostrucutres. Intrinsic relevant defects can be created in the material from the single to the ensemble level with electron irradiation.

$\mathrm{SiC}$ nanoparticles also harbour stable intrinsic defects with single photon emitter, therefore we believe that this will be a rich platform for future investigation towards technological advanced systems and novel physics to be discovered.

\section{REFERENCES}

[1] Madar, R., "Materials science: Silicon carbide in contention," Nature 430, 974-975 (2004).

[2] DiVincenzo, D. , "Quantum bits: Better than excellent," Nature Materials 9, 468-469 (2010).

[3] Koehl, W. F. , Buckley, B. B. , Heremans, F. J. , Calusine, G. and Awschalow, D. D. , "Room temperature coherent control of defect spin qubits in silicon carbide," Nature 479, 84 (2011).

[4] Smart, A. G. "'Silicon carbide defects hold promise for device-friendly qubits," Physics Today 65(1), 10-11 (2012).

[5] Castelletto, S., Johnson, B. C., Ivády, Stavrias, V. N., Umeda, T. , Gali , A. and Ohshima, T. "AA silicon carbide room-temperature single-photon source," Nature Materials in press DOI: 10.1038/NMAT3806

[6] S. Castelletto, B. C. Johnson, A. Boretti, "Quantum effects in silicon carbide hold promise for novel integrated devices and sensors," Advanced Optical Materials, 1, 609-625 (2013). 
[7] Umeda, T., Son, N. T. ,Isoya, Janzen, J. E. , Ohshima, T., Morishita, N., Itoh, H., Gali, A. and Bockstedte, M., "Identication of the carbon antisite-vacancy pair in 4h-sic," Phys. Rev. Lett. 96,145501 (2006).

[8] Steeds, J. W., "Photoluminescence study of the carbon antisite-vacancy pair in 4h- and 6h-sic," Phys. Rev. B $80,245202(2009)$.

[9] Fan, J., Li, H. , Wang, J. ,and Xiao, M. ,"Fabrication and photoluminescence of sic quantum dots stemming from 3C, 6H, and 4H polytypes of bulk SiC," Applied Physics Letters 101(13), 131906 (2012).

[10] Magyar, A. P., Aharonovich, I. , Baram, M., and Hu, E. L., "Photoluminescent SiC tetrapods", Nano Letters 13, $1210-1215$ (2012).

[11] Johnson, B.C. , Iwamoto, N. , Castelletto, S., Onoda, S. , Ohshima, T. , Karle, T. and McCallum, J. C., “Optically active defect centres in silicon carbide devices" in preparation.

[12] S. Castelletto, B.C. Johnson "Room temperature single photon emission from 3C silicon carbide nanoparticles", in preparation.

[13] S. Castelletto, A. P. Magyar, I. Aharonovich , “ Room temperature Observation of non- classical emission from semiconductor quantum confined excitons in silicon carbide tetrapods", (in preparation). 